\title{
Charles Bonnet et Vincenzo Malacarne sur le cervelet siège de l'âme et sur l'impression basilaire du crâne dans le crétinisme
}

Par Luigi Belloni

Facultati Medicae Genevensi Almae Matri tamquam suae

Dans ce colloque historique dédié à «Genève et la médecine», ma contribution voudrait souligner, par un exemple moins connu, le rôle de conscience critique de la biologie, même de la biologie médicale, exercé au XVIII ${ }^{\mathrm{e}}$ siècle par le genevois Charles Bonnet (1720-1793) ${ }^{1}$. Devenu pratiquement aveugle après un exorde expérimental brillant, et obligé, de ce fait, à abandonner les recherches de laboratoire, Bonnet se concentra dans la biothéorétique, dans des ouvrages de psychologie, philosophie et théologie, et dans un échange actif avec des savants insignes de l'époque, en inspirant leurs recherches et en discutant subtilement leurs résultats. Ainsi, sa correspondance constitue une source de premier ordre pour l'Histoire de la Science au XVIII ${ }^{\mathrm{e}}$ siècle.

Par rapport à l'importance de Haller ou de Spallanzani, celle du correspondant dont nous nous occupons, Michele Vincenzo Giacinto Malacarne (1744-1816) ${ }^{2}$, est certainement bien différente. Exerçant la chirurgie à Acqui de 1775 à 1783 et à Turin de 1783 à 1789, Malacarne est surtout connu comme neuroanatomiste et, en particulier, comme auteur de la Encefalotomia nuova universale (Turin 1780) ${ }^{3}$. Grâce à l'amabilité de Monsieur Ph. Monnier, conservateur du Département des Manuscrits de la Bibliothèque Publique et Universitaire de Genève, j'ai pu étudier sur les xérocopies toute la correspondance Bonnet-Malacarne: 53 lettres $^{4}$, de 1778 jusqu'à 1789, l'année même au cours de laquelle Malacarne quitta Turin pour Pavie, appelé à la chaire d'Institutions Chirurgicales et d'Obstétrique de l'Université de cette ville. Les deux correspondants se rencontrèrent une seule fois, à Genthod, durant l'été de $1785^{5}$.

Transféré en 1775 comme chirurgien dans une petite ville telle qu'Acqui, Malacarne eut toutefois la possibilité de continuer à effectuer les anatomies humaines qu'il faisait auparavant au collège chirurgical de Turin : et il se servit largement de cette possibilité pour effectuer des recherches neuroanatomiques qu'il étendit également aux animaux inférieurs et surtout aux oiseaux.

En 1776 il publia à Turin la Nuova esposizione della vera struttura del cervelletto umano $^{6}$, fondée sur l'anatomie de 44 cas.

Le 8 Août 1778 il entra en contact épistolaire avec Bonnet ${ }^{7}$, et il reconnu, avant tout, ce qu'il lui devait pour avoir puisé l'inspiration de ses recherches dans des 
ouvrages tels que l'Essai analytique sur les facultés de l'âme $(1760)^{8}$ et, encore davantage, l'Analyse abrégée de l'Essai analytique (1764) ${ }^{9}$.

Je n'ai pas l'intention, ni même la compétence pour traiter de façon convenable la psychologie, typiquement sensualiste, de Bonnet ${ }^{10}$. Le temps à ma disposition m'oblige en outre à me limiter au point fondamental de la correspondance : le problème de la jonction somato-psychique, que René Descartes (1596-1650), en abolissant les esprits traditionnels de notre organisme ${ }^{11}$, avait reconnu dans la glande pinéale (épiphyse). Cette jonction, appelée plus couramment par les anatomistes «siège de l'âme», était toutefois destinée à subir de nombreux transferts: par exemple, dans le corps calleux, selon François de La Peyronie (16781747 $)^{12}$, et Giovanni Maria Lancisi $(1654-1720)^{13}$; et dans le bulbe de l'encéphale (moelle allongée), selon Anne-Charles Lorry (1726-1783) ${ }^{14}$ en 1760. Bonnet, lui, ne reconnaissait pas une partie bien définie du cerveau comme siège de l'âme ; mais il assignait à l'anatomie la tâche de la localiser dans le lieu de confluence des extrémités des nerfs des sens («sensorium») : une position assez proche de celle d'Albrecht von Haller (1708-1777), qui reconnaissait le siège de l'âme génériquement dans la «medulla cerebri». ${ }^{15}$

Dans l'Essai d'application des principes psychologiques $(1769)^{16}$, Bonnet est même franchement pessimiste : "Nous sommes fort peu éclairés sur la structure intime du Cerveau! L'Anatomie se perd dans ce Dédale ténébreux. [...] Nous devons donc renoncer à déterminer précisément quelle est la partie du Cerveau qui constitue le Siège de l'Ame.»

Pour atténuer ce scepticisme, Malacarne ${ }^{17}$ propose l'adoption d'un procédé de recherche proche de la méthode anatomo-clinique: pendant la vie, observation «du tempérament, des penchants, de la vivacité, du talent, de la tendance à certaines infirmités» de chaque sujet; après la mort, examen anatomique approfondi des différentes parties de l'encéphale, de façon à établir des parallélismes anatomo-psychiques, fondés naturellement sur une vaste casuistique. Il s'agit au fond d'une recherche d'organologie dans le sens de Franz Joseph Gall (17581828) ${ }^{18}$, qui établira un parallélisme entre 27 organes répandus dans l'encéphale et autant de facultés de l'âme conçues comme des fonctions de ces mêmes organes.

Au cours de la correspondance, Bonnet pousse Malacarne à déterminer «la partie du cerveau qui est le siège ou l'instrument le plus immédiat des opérations de notre âme ", ${ }^{19}$ c'est-à-dire à reconnaître dans l'encéphale le lieu de confluence des extrémités des nerfs des sens. Malacarne constate par contre que les nerfs des sens, au lieu de converger, s'écartent toujours plus au fur et à mesure qu'ils s'enfoncent dans le cerveau. Sur quoi Bonnet avance l'hypothèse selon laquelle la divergence serait suivie d'une convergence invisible à l'œil nu : et Malacarne 
rappelle, à ce propos, le comportement analogue du double arbre veineux du foie (divergence des rameaux de la veine porte et convergence des rameaux des veines hépatiques).

Malacarne toutefois suit une autre piste pour reconnaître le siège de l'âme : et cela dès la première lettre ${ }^{20}$, dans laquelle il rappelle l'hypoplasie du cervelet, avec une forte réduction du nombre de ses lamelles, qu'il avait observée dans un imbécile disséqué en 1775 («le fat de Morsasco ») ${ }^{21}$ : une altération qu'il observera à nouveau, et d'une façon essentiellement identique, chez deux autres imbéciles disséqués en $1779^{22}$ et en $1782^{23}$. Ayant établi le parallélisme anatomo-psychique entre hypoplasie du cervelet et imbécillité, il s'ensuit que le cervelet est le siège de l'âme.

L'hypoplasie du cervelet est secondaire à une malformation du crâne que nous appelons d'après Rudolf Virchow (1821-1902) ${ }^{24}$ "impression basilaire » et qu'aussi les praticiens ont appris à connaître grâce aux progrès de la neuroradiologie ${ }^{25}$ et des disciplines corrélatives. L'interprétation de l'altéxation du crâne observée par Malacarne est avant tout fondée sur la description qu'il fit dans la Lettre sur l'état des Crétins ${ }^{26}$ adressée vers la fin de 1788 à Johann Peter Frank (1745-1821), alors professeur de Clinique Médicale à l'Université de Pavie :

1. Le crâne en général moins élevé, et moins applati sur les côtés.

2. Les trous du Valsalva ${ }^{27}$ aux angles Lambdoïdiens beaucoup plus grands; et au contraire

3. Les trous déchirés à la base du crâne, entre l'apophyse basilaire de l'occipital, et les rochers des os temporaux, presque oblitérés, de façon qu'à peine peuvent-elles passer par les ouvertures de ces mêmes trous, les paires des nerfs sympathiques moyens, des Glosso-pharyngiens, et de l'Accessoire du Willis : ce qui rend

4. Les sinus latéraux de la Dure-mère beaucoup plus amples dans toute leur extension, et

5. La tente du cervelet beaucoup trop épaisse : conséquemment

6. Le cervelet lui-même logé dans une cavité plus étroite qu'il ne lui faut, ne pouvant pas se développer, ni acquérir la grosseur, et la largeur ordinaire, doit porter sur les fonctions animales les atteintes, et sur certains organes extérieurs les dérangemens qu'on n'a que trop lieu d'observer chez ces malheureux.

7. Il a rẹmarqué aussi chez les trois imbécilles, que l'apophyse basilaire de l'occipital au lieu de se porter en avant avec une douce obliquité en haut (depuis les apophyses condiloïdiennes du même os, duquel le grand trou est situé naturellement horizontalement), fait un plan presque de niveau avec les apophyses clinoïdiennes du sphénoïde ; et le grand trou s'ouvre, non comme à l'ordinaire de haut en bas, mais d'en arrière en avant, ce qui fait

8. Que la masse du cervelet infiniment plus gênée, reste toujours moindre à proportion des entraves opposées à son accroissement, par la mauvaise conformation des os.

9. Le nombre des feuillets lamineux, et des lames qui en composent la plus grande partie, n'y est pas égal comme aux autres hommes. [Et c'est ce qu'on prie avec ardeur Messieurs les Chirurgiens de vouloir bien vérifier; puisque de ce fait bien démontré ne peut que résulter nombre de connaissances très-utiles pour l'explication de plusieurs 
phénomènes qui intéressent en dernier ressort l'économie animale, et l'explication des facultés attachées aux organes contenus dans les trois cavités principales de notre corps $]^{28}$.

10. Enfin la moëlle allongée doit se courber en gagnant le canal de l'épine par le grand trou occipital d'une façon très-préjudiciable aux nerfs qui en sortent; et les fonctions de ces nerfs, et des dépendances de toute la moëlle épinière, se déranger.

Encore plus probants sont les deux dessins (fig. 1 et 2) parus en 1795 avec la Beschreibung der beiden Cretinen-Schedel in Pavia, von Hrn. Hospital-Medicus Dr. Michaelis ${ }^{29}$, adressée à Johann Friedrich Blumenbach $(1752-1840)^{30}$, un des pères de l'anthropologie moderne. Il s'agit des crânes de deux des trois imbéciles que Malacarne avait envoyés à Frank en même temps que la Lettre sur l'état des crétins et que Frank avait déposés au Musée Pathologique agrégé à sa Clinique.

Dans les deux dessins exécutés par Michaelis «nach der Natur in Camperscher ${ }^{31}$ Manier mit wanderndem Gesichtspuncte », ${ }^{32}$ la ligne pointillée correspond au profil de la section sagittale médiane de la base du crâne, et démontre le caractère de l'anomalie : «comme si la zone environnant le grand trou occipital était enfoncée vers l'intérieur de la cavité du crâne ${ }^{33}{ }^{33}$ 'où le rapetissement sensible de la fosse crânienne postérieure et la conséquente hypoplasie du cervelet.

Des fortes réserves furent avancées par Bonnet sur le parallélisme entre imbécillité et hypoplasie du cervelet, laquelle, en raison même de la casuistique peu nombreuse, pourrait être une simple altération collatérale, et non pas fondamentale. Bonnet suggéra d'effectuer des expériences pour démontrer que l'exercice continu et intense de la fonction comporte le développement de l'organe, bien que leur valeur probante ne soit pas absolue : «Le travail de l'esprit peut augmenter le nombre des lamelles. Il est au moins très sûr qu'il fait affluer le sang au cerveau » et, par conséquent, «le nombre des lamelles dépendrait de l'exercice de la capacité intellectuelle», et non pas le contraire ${ }^{34}$.

Le 23 Mars 1782 Malacarne envoya les données qu'il avait recueillies et élaborées en tabelle ${ }^{35}$ d'après une vaste casuistique, en considérant pour chaque sujet le rapport pondéral cerveau-cervelet et le nombre des lamelles du cervelet. Bonnet suggéra d'étendre l'étude numérique comparée des lamelles du cervelet également aux singes et, surtout, aux orang-outangs: Malacarne ne réussit toutefois à disséquer qu'un macaque et un cynocéphale ${ }^{36}$.

Pour remédier à l'insuffisance de la casuistique que lui avait fait remarquer Bonnet, Malacarne envisagea l'opportunité de continuer ses recherches en vallée d'Aoste, «où l'on me dit qu'il y a des paroisses entières de ces sortes d'imbéciles ». ${ }^{37}$ Ainsi écrivit-il en 1782 à Bonnet qui, en partie inséra cette lettre, et en partie la résuma et la commenta, dans la réédition de la Palingénésie philoso- 

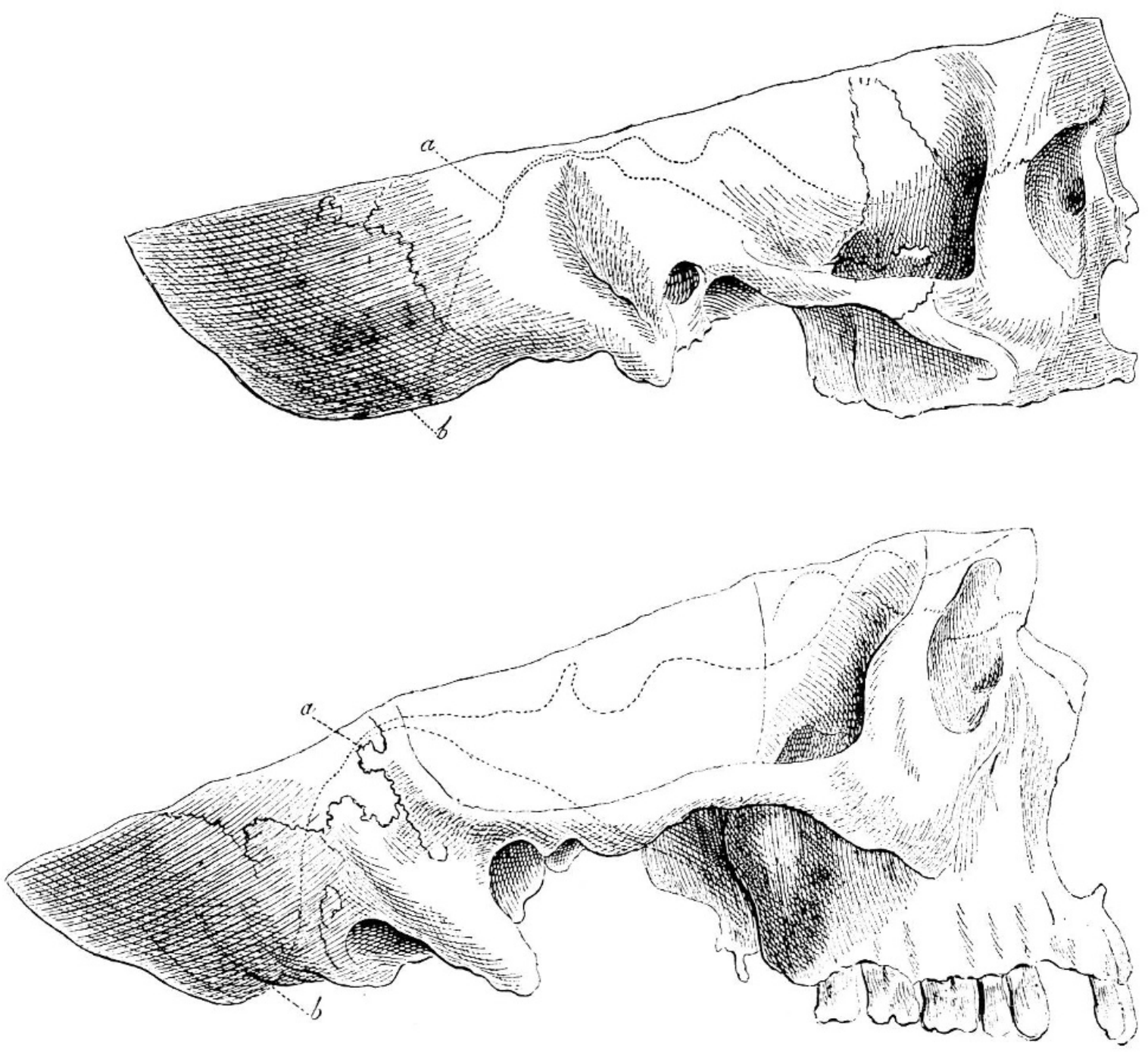

Figures 1 et 2. Reproduction en dimensions réduites (grossissement $0,63 \times$ ) des deux figures accompagnant la Beschreibung der beiden Cretinen-Schedel in Pavia, von Hrn. HospitalMedicus Dr. Michaelis publiée par Johann Friedrich Blumenbach dans la Medicinische Bibliothek 3 (1795), p. 664-668. Pour faciliter la comparaison, et pour des raisons d'esthétique, la figure de la table II a été reproduite au dessus de la figure de la table I.

La ligne pointillée correspond au profil de la section sagittale médiane de la base du crâne, et démontre le caractère de l'anomalie : "Am auffallendsten ist die Basis verändert, die einen Druck von außen nach innen erlitten hat, deren Mittelpunct ungefähr der vordere Rand (-a-) des großen Lochs $(-a . b-)$ ist, und eine mäßige Faust aufnimmt. » D'où le rapetissementde la fosse crânienne postérieure et la conséquente hypoplasie du cervelet.

Le crâne complètement édenté appartient au «fat de Morsasco » disséqué en 1775. Cette identification est déduite d'après une comparaison faite par Malacarne lui-même dans l'Auctarium observationum et iconum ad osteologiam et osteopathologiam VV. CC. C. G. Ludvigii et Antonii Scarpae, Patavii 1801, pages 110-111 et Note (a) à la page 110. 
phique $^{38}$, en ajoutant à son tour, le conseil d'étendre les recherches aux «Cretins du Valais et des Vallées voisines $»{ }^{39}$ conseil qu'il renouvellera à Malacarne en $1786^{40}$.

Rappelons ici que le terme cretin propre du dialecte valaisan, dépassa les frontières de la vallée pour acquérir une diffusion générale grâce à l'article, intitulé justement Cretins, inséré en 1754 par Jean d'Alembert (1717 env.-1783) dans l'Encyclopédie ${ }^{41}$. En 1763 A. von Haller donna une description brève, mais complète, des crétins du Valais ${ }^{42}$, et revint en 1770 sur le sujet, en parlant du climat de $R_{o c h}{ }^{43}$. En 1786 suivit la description contenue dans le $2^{\text {ème }}$ volume des Voyages dans les Alpes ${ }^{44}$ d'Horace-Bénédict de Saussure (1740-1799), qui avait effectué ses premières observations sur un groupe de crétins à Villeneuve en Vallée d'Aoste. Neveu et élève de Bonnet, de Saussure connaissait les altérations cranioencéphaliques observées par Malacarne et réitéra le conseil de son oncle à ce qu'ils soient contrôlés sur une plus nombreuses casuistique ${ }^{45}$.

D'Alembert, Haller et de Saussure décrivirent dans les crétins des vallées alpines deux éléments très importants - le goitre et le caractère endémique - qui n'étaient pas présents dans la description des trois imbéciles de Malacarne.

Les temps étaient désormais mûrs pour soumettre à la recherche anatomique les crétins des Alpes. La lettre de Bonnet, datée du 13 Novembre $1787^{46}$, que Malacarne fit tomber sous les yeux du comte Francesco Baldassarre di Perrone, Ministre des affaires Etrangères du Royaume de Sardaigne ${ }^{47}$, fournit l'occasion pour que Malacarne fût chargé de rédiger le Mémoire adressé à Messieurs les Chirurgiens de la Vallée d'Aouste, et des Provinces voisines, pour en avoir des notions exactes des parties extérieures et internes du cou, et de la tête des Crétins ${ }^{48}$. Le 18 Novembre 1787, le Mémoire était déjà prêt et Perrone se chargea de sa transmission par voie officielle; mais nous ignorons s'il obtint des réponses de valeur essentielle ${ }^{49}$, d'autant plus qu'en 1789 Malacarne quitta Turin pour la chaire de Pavie. Ce Mémoire, qui contient la description en 10 points des altérations du crâne et de l'encéphale, est compris à son tour dans la Lettre sur l'état des Crétins qui fut publiée, non seulement par Frank ${ }^{50}$, mais aussi par Malacarne et, avec le texte en italien ${ }^{51}$, dans la brochure $S u$ i gozzi e sulla stupidità che in alcuni paesi gli accompagna Tentativi di V. Malacarne... (Turin 1789) ${ }^{52}$.

Malacarne suscita un intérêt considérable, aussi bien par sa description, que par les deux crânes (fig. 1 et 2) qu'il donna à Frank et que celui-ci offrit à l'admiration des jeunes médecins allemands qui accouraient nombreux pour suivre ses leçons. Rappelons en particulier: Jakob Fidelis Ackermann (1765-1815) de Mayence, auteur du livre Über die Kretinen, eine besondre Menschenabart in den Alpen (Gotha 1790) ${ }^{53}$; Gottfried Philipp Michaelis ${ }^{54}$ de Göttingen, auteur de la 
Beschreibung der beiden Cretinen-Schedel in Pavia (1795) contenant les deux dessins que nous avons déjà vus (fig. 1 et 2) ; les frères Joseph Wenzel (1768-1808) et Karl Wenzel (1769-1827) de Mayence, auteurs du livre Über den Cretinismus (Wien 1802) ${ }^{55}$.

A Turin parut, en 1792, l'Essai sur le goître et le crétinage ${ }^{56}$ de François-Emanuel Foderé (1764-1835), qui était particulièrement intéressé au problème du crétinisme, étant né à Saint-Jean de Maurienne ${ }^{57}$. Fodéré nia, de la façon la plus nette, l'importance centrale que Malacarne avait attribuée au cervelet: «Je me sens obligé de dire ici que je ne crois pas que son travail ait jeté quelque jour sur une cause aussi obscure. $\aleph^{58}$

Ce sera Virchow, vers la moitié du $\mathrm{XIX}^{\mathrm{e}}$ siècle, qui détachera définitivement l'impression basilaire du cadre du crétinisme endémique ${ }^{59}$ et qui lui attribuera par contre une signification anthropologique ${ }^{60}$. Dans le crétinisme il souligna, au contraire, une autre malformation du crâne, et précisément le raccourcissement de la base, qu'il attribuait à la synostose précoce de la synchondrose sphénooccipitale ${ }^{61}$. Sa doctrine contenait en même temps un progrès et une erreur.

De même que Malacarne s'était trompé en plaçant l'origine du crétinisme dans une malformation du crâne qui, bien que constante dans ses trois imbéciles, était simplement une altération collatérale; ainsi Virchow observa la synostose précoce de la base crânienne à la dissection d'un nouveau-né ${ }^{62}$, qu'il jugeait crétin, mais dans lequel les recherches histologiques du psychiatre Wilhelm Weygandt (1870-1939) ${ }^{63}$, reconnurent en 1904 un cas de chondrodystrophie fœtale. Par Weygandt même, et aussi par d'autres auteurs avant et après lui ${ }^{64}$, le raccourcissement de la base crânienne des crétins fut expliqué, non pas par la synostose précoce, mais par l'ossification retardée de la synchondrose sphéno-occipitale qui reste longtemps persistante après le terme de la croissance.

A travers ses vicissitudes et ses fausses routes, l'histoire de la craniologie dans le crétinisme offre un des nombreux exemples de la valeur formative de l'Histoire de la Médecine. Mais aujourd'hui je désire particulièrement souligner la part très importante prise par les savants genevois :

Charles Bonnet qui, par ses écrits de psychologie, provoqua les recherches de Malacarne et, dans ses lettres à lui, les discuta et en partie les dirigea ;

Horace-Bénédict de Saussure qui, par sa description classique, apporta une contribution fondamentale à ce que Paul F. Cranefield ${ }^{65}$ appela «the definitive discovery of cretinism ». 


\section{Notes}

${ }^{1}$ Raymond Savioz, Mémoires autobiographiques de Charles Bonnet de Genève, Paris 1948 ; La philosophie de Charles Bonnet de Genève, Paris 1948.

${ }^{2}$ La principale bibliographie sur Malacarne est rapportée par: Giuseppe Ongaro, Les apports de Vincenzo Malacarne (1744-1816) à la tératologie, Verhandlungen des XX. Internationalen Kongresses für Geschichte der Medizin, Berlin 1966, Hildesheim 1968, p. 186194, avec portrait (v. note 1 à p. 192).

${ }^{3}$ En trois parties, dont chacune a sa propre pagination. La première partie est consacrée au crâne, aux méninges et aux sinus veineux; la seconde au cerveau; la troisième au cervelet. Cette dernière avait déjà été publiée, en forme indépendante, en 1776 (v. l'ouvrage rappelé par la note 6). Pour des notices sur un manuscrit autographe de Malacarne qui servit pour la rédaction de cet ouvrage, voir: Luigi Castaldi, Un manoscritto di Vincenzo Malacarne saluzzese sull'anatomia delle meningi, Rivista di Storia delle Scienze Mediche e Naturali 19 (1928), p. 62-73.

Comme quatrième partie de l'Encefalotomia nuova universale fut conçu le traité Sull'origine de' Nervi i quali escono dalla base del cranio, dédié à Bonnet en 1779 et publié dans la Nevro-encefalotomia, Pavia 1791, p. 58-217.

${ }^{4}$ Ainsi distribuées : 27 lettres de Malacarne à Bonnet (Mss. 34, 36, 37, 39, 40); 20 lettres de Bonnet à Malacarne (Mss. 75, 76, 77); 2 lettres de Jannette Mayan (ou Maillan), épouse de Malacarne, à Bonnet ; 3 lettres de Bonnet à Jannette Mayan Malacarne. Une autre lettre de Malacarne à Bonnet, du 26 Janvier 1787, se trouve dans l'Arch. de Saussure $\mathrm{N}^{\circ} 11$.

Rappelons brièvement les éditions auxquelles les deux correspondants soumirent les premières lettres de leur correspondance. Malacarne publia les 5 premières lettres de Bonnet, toutes datées de 1779 , en appendice à la troisième et dernière partie de l'Encefalotomia nuova universale (1780); et Bonnet les fit bientôt réimprimer dans les Euvres d'histoire naturelle et de philosophie de Charles Bonnet 12 (1781), p. 451-483. Des extraits et des passages de lettres de Malacarne, toujours relatifs à notre sujet, furent insérés par Bonnet dans les mêmes Euvres 15 (1783), p. 130-135, 205-209. Dans les présentes et dans les suivantes citations des Euvres, nous indiquons la tomaison et la pagination selon l'édition in-8.

Les 7 premières lettres de Malacarne et les 7 lettres correspondantes de Bonnet, traduites en Italien par Malacarne, parurent dans la Biblioteca fisica d'Europa 13-19 (1790/91) et furent par la suite recueillies par Malacarne dans le volume Nevro-encefalotomia, Pavia 1791.

${ }^{5}$ Lettre de Bonnet à Jannette Malacarne, datée du 20 Septembre 1785 (Ms. 76, c. 222 r).

${ }^{6}$ Voir la Note 3.

${ }^{7}$ Ms. 34, cc. 172-175 (lettre autographe en italien) et 176-178 (traduction en français).

${ }^{8}$ Nouvelle édition dans Euvres 13-14, Neuchâtel 1782.

${ }^{9}$ Déjà comprise dans la préface à la Contemplation de la Nature (1764), fut ensuite mise comme préface à La Palingénésie philosophique (1769). Réimpression dans Euvres 15 (1783), p. 1-58.

L'Essai de Psychologie, publié anonyme à Leyde en 1754, fut inséré par Bonnet dans ses Euvres 17 (1783), p. 1-235. Voir Jean Starobinski, L'Essai de Psychologie de Charles Bonnet : Une version corrigée inédite, Gesnerus 32 (1975), p. 1-15. 
10 Voir le second ouvrage de Savioz cité à la Note 1.

${ }^{11}$ Luigi Belloni, "Rete mirabile» (Introduzione storica). Symposium International sur le diencéphale (Milan 3-5 Mai 1956), Pathophysiologia Diencephalica, Wien 1958, p. 3-17.

12 De La Peyronie, Observations par lesquelles on tâche de découvrir la partie du Cerveau où l'Ame exerce ses fonctions, Histoire de l'Académie Royale des Sciences. Année MDCCXLI. Avec les Mémoires de Mathématique \& de Physique, pour la même Année. Tirés des Registres de cette Académie, Paris 1744, p. 39-45 de l'Histoire et p. 199-218 des Mémoires.

${ }^{13}$ Felice Grondona, La dissertazione di Giovanni Maria Lancisi sulla sede dell'anima razionale, Physis 7 (1965), p. 401-430.

${ }^{14}$ Lorry, Sur les mouvemens du cerveau et de la dure-mère. - Premier Mémoire, Sur le mouvement des parties contenues dans le crâne, considérées dans leur état naturel. Second Mémoire, Sur les mouvemens contre nature de ce viscère, et sur les organes qui font le principe de son action, Mémoires de mathématique et de physique présentés à l'Académie Royale des Sciences, par divers Savans, et lûs dans ses Assemblées 3 (1760), p. IX-X, 277-313, 344-377.

${ }^{15}$ Elementa physiologiae corporis humani 4, Lausanne 1762, p. 393 (Lib. X : Cerebrum et nervi, Sectio VIII: Conjecturae, §XXIII : Animae sedes: «Nunc et sensus et motus in cerebri medulla scaturiginem suam habent. Erit ergo ea animae sedes.»).

Particulièrement éloquents apparaissent certains passages des lettres de von Haller à Bonnet insérés par ce dernier dans sa lettre à Malacarne du 12 Mai 1779 (Ms. 75, c. 194).

${ }^{16}$ Charles Bonnet, Essai d'application des principes psychologiques de l'Auteur à la manière dont les idées sont rappellées par les mots, et à l'association des idées en général en préface à La Palingénésie philosophique (1769). Réimpression dans Euvres 15 (1783), p. 129.

${ }^{17}$ Vincenzo Malacarne, Nuova esposizione della vera struttura del cervelletto umano, Torino 1766, p. 13-14.

${ }^{18}$ Sur l'influence de Bonnet sur Gall, voir Erna Lesky, Structure and function in Gall, Bulletin of the History of Medicine 44 (1970), p. 297-314; Georges Lanteri-Laura, Histoire de la Phrénologie. L'homme et son cerveau selon F.J.Gall, Paris 1970, p. 54 ss.

${ }^{19}$ Lettre de Bonnet à Malacarne, du 3 Septembre 1779 (Ms. 75, c. 217r).

${ }^{20}$ Rappel de la Note 7.

${ }^{21}$ Vincenzo Malacarne, Encefalotomia nuova universale, Torino, $1^{\text {re }}$ Partie, p. 106.

${ }^{22}$ Lettre de Malacarne à Bonnet, du 11 Décembre 1779 (Ms. 36, c. 1r).

${ }^{23}$ Lettre de Malacarne à Bonnet, du 23 Mars 1782 (Ms. 36, c. 19r).

${ }^{24}$ Rudolf Virchow, Beiträge zur physischen Anthropologie der Deutschen, mit besonderer Berücksichtigung der Friesen, Abhandlungen der K. Akademie der Wissenschaften zu Berlin. Aus dem Jahre 1876, Physikalische Klasse, 1. Abtheilung, Berlin 1877, p. 1-391 et tables I-V (voir p. 317 ss.).

Aux ouvrages du Maître ne tarda pas à suivre la contribution de Paul Grawitz (18501932), à l'époque assistant à Berlin, et par la suite Professeur d'Anatomie Pathologique à Greifswald : Beitrag zur Lehre von der basilaren Impression des Schädels, [Virchow's] Archiv für pathologische Anatomie und Physiologie und für klinische Medicin 80 (1880), p. 449-474 et table XIII.

${ }^{25}$ Pour les premières communications radiologiques, voir Arthur Schüller, Die Schädelbasis im Röntgenbilde (= Fortschritte auf dem Gebiete der Röntgenstrahlen, Ergänzungs- 
band 11), Hamburg 1905, p. 53-55 et figures 1 et 2 de la table V; Zur Röntgen-Diagnose der basalen Impression des Schädels, Wiener Medizinische Wochenschrift (1911), colonnes 2593-2599 ; ce dernier article fut repris par le même auteur dans sa monographie Röntgendiagnostik der Erkrankungen des Kopfes, Wien 1912.

Le neuropsychiatre Arthur Schüller (1874-1958) fut l'un des pionniers de la radiologie médicale dans l'orbite de Guido Holzknecht (1872-1931) à Vienne.

${ }^{26}$ Lettre de Monsieur Vincent Malacarne... au Professeur Frank à Pavie sur l'état des crétins. De Turin au mois de Décembre 1788. Insérée par Frank lui-même dans un choix d'opuscules, surtout d'auteurs allemands, dont il s'occupait de la publication à l'époque : Delectus opusculorum medicorum antehac in Germaniae diversis Academiis editorum. Quae in Auditorum Commodum collegit, et cum notis hinc inde aucta recudi curavit J.P.F. ... 6, Ticini 1789, p. 241-258. Le don des crânes au Musée Pathologique de Pavie est signalé dans une note au bas de la page.

${ }^{27}$ Dans le De aure humana tractatus (Bologna 1704) de Anton Maria Valsalva (1666-1723) est signalée "vena, adhuc ab aliis non descripta, quam ego occipitalem appellabo» (p. 14 et Fig. II de la table VII), laquelle «per peculiare novum foramen intra cranium ingreditur » et s'ouvre dans le sinus latéral (c'est la veine aujourd'hui couramment appelée «émissaire mastoïdien»). L'orifice du «novum foramen», à la surface externe du crâne, est indiqué par $d . d$. dans la table IV du traité de Valsalva.

${ }^{28} \mathrm{~J}$ 'ai mis entre crochets la dernière partie du point 9, étant donné son caractère de recommandation (aux chirurgiens de la Vallée d'Aoste auxquels la description s'adresse; voir le passage rappelé par les Notes 49 et 50 ).

${ }^{29}$ Dans le Lexikon de Hirsch, le Dr. Michaelis, auteur de la Beschreibung, n'a pas un article à lui, mais il est vraisemblablement possible de l'identifier dans Gottfried Philipp M. cité à l'article «Gustav Adolf Michaelis», son fils, né à Harburg en 1798. En 1796, il se présente en effet comme "Garnisonsarzt zu Haarburg» dans l'article: Ph. Michaelis, Über einen gelben Fleck und ein Loch in der Nervenhaut des menschlichen Auges, Journal der Erfindungen, Theorien und Widersprüche in der Natur- und Arzneiwissenschaft (Gotha), XV. Stück (1796), p. 3-17, où l'auteur rappelle un séjour qu'il fit à Pavie et des observations effectuées à Pavie par ses amis Wenzel de Mayence. Une lettre successiv'; du même Michaelis, datée de Haarburg, le 22 Avril 1796, parut sur la même revue (XVII. Stück, 1796, p. 121-133). Voir Luigi Belloni, Francesco Buzzi e la scoperta della macula lutea, L'Ospedale Maggiore 44 (1956), p. 223-234.

A page 2 de l'ouvrage Über den Cretinismus, cité à la Note 55, les frères J. et K. Wenzel rappellent la «Fußreise» qu'ils firent pour étudier les crétins de la région de Salzburg «im Jahre 1792 in Begleitung des Herrn Doctor Michaelis von Göttingen». Le même voyage en compagnie des frères Wenzel est cité par Michaelis également dans l'article: Ủber die Cretinen im Salzburgischen, Medicinische Bibliothek 3, Viertes Stück (1795), p. 640-663 (voir p. 641).

${ }^{30}$ Johann Friedrich Blumenbach, professeur de Médecine à l'Université de Göttingen, publia la description et les dessins dans une revue éditée par lui dans la même ville : Medicinische Bibliothek 3, Viertes Stück (1795), p. 664-668, tab. I et II.

${ }^{31}$ Dissertation sur les variétés naturelles qui caractérisent la physionomie des hommes des divers climats et des différens âges. Suivie de Réflexions sur la Beauté; particulièrement sur celle de la tête; avec une Manière nouvelle de dessiner toute sorte de têtes avec la plus grande 
exactitude. Ouvrage posthume de M. Pierre Camper. Traduit du Hollandois par H. J. Jansen. Paris/La Haye 1791.

Dissertation physique de Mr. Pierre Camper, sur les différences réelles que présentent les traits du visage chez les hommes de différents pays et de différents âges; sur le beau qui caractérise les statues antiques et les pierres gravées. Suivie de la proposition d'une nouvelle méthode pour dessiner toutes sortes de têtes humaines avec la plus grande sûreté. Publiée après le Décès de l'Auteur par son fils Adrien Gilles Camper. Traduite du Hollandois par Denis Bernard Quatremère D'Isjonval. Utrecht 1791.

Peter Camper über den natürlichen Unterschied der Gesichtszüge in Menschen verschiedener Gegenden und verschiedenen Alters; über das Schöne antiker Bildsäulen und geschnittener Steine; nebst Darstellung einer neuen Art, allerlei Menschenköpfe mit Sicherheit zu zeichnen. Nach des Verfassers Tode herausgegeben von seinem Sohne Adrian Gilles Camper. Übersetzt von S.Th. Sömmerring. Berlin 1792.

${ }^{32}$ Page 56 de l'ouvrage des frères Wenzel rappelé par la Note 55.

${ }^{33}$ «... bei welchem eine eigentümliche Veränderung des Schädelgrundes, namentlich eine Art von Eindrückung der Umgebung des Hinterhauptsloches gegen den Schädelraum stattfindet. 》 (page 317 de l'ouvrage de R. Virchow, cité à la Note 24.)

${ }^{34}$ Lettre de Bonnet du 24. Décembre 1779 (Ms. 75, c. 229r).

${ }^{35}$ Ms. 36, cc. 21r et 22r. La tabelle fut ensuite insérée par Malacarne dans le traité Delle osservazioni in chirurgia 2, Torino 1784, p. 86-89.

${ }^{36}$ Lettre de Malacarne du 29 Août 1786 (Ms. 39, c. 154v).

${ }^{37}$ Lettre de Malacarne du 23 Mars 1782 (Ms. 36, c. 19).

${ }^{38}$ Euvres 15, p. 208-209.

39 «Les Cretins du Valais et des Vallées voisines procureraient en ce genre de riches moissons aux Physiologistes qui voudraient approfondir un Sujet si digne de l'être.»

${ }^{40}$ Lettre de Bonnet du 16 Septembre 1786 (Ms. 77, c. 38).

${ }^{41}$ Encyclopédie, ou Dictionnaire raisonné des Sciences, des Arts et des Métiers 4 (1754), p. 459.

${ }^{42}$ Albrecht von Haller, Elementa physiologiae corporis humani 5, Lausanne 1763, p. 570-574 (Liber XVII : Sensus interni, Sectio I : Intellectus, Caput XVII : Fatuitas).

43 Albrecht von Haller, Commentatio de vento Rupensi, Prael. d. VIII. Dec. MDCCLXX., Novi Commentarii Societatis Regiae Scientiarum Gottingensis ad A. MDCCLXIX. et MDCCLXX. 1 (1771), p. 30-44 (voir p. 43-44).

${ }^{44}$ Horace-Bénédict de Saussure, Voyages dans les Alpes, précédés d'un essai sur l'histoire naturelle des environs de Genève 2, Genève 1786, p. 480-488, §§ 1030-1036 du chap. XLVII «Des crétins et des albinos».

45 «Il seroit bien intéressant de réaliser le souhait de M. Bonnet, et de répéter sur les Crétins les belles observations qu'a faites M. Malacarne sur les cerveaux de quelques imbécilles. Il faudroit qu'un habile anatomiste disséquât leur cerveau, et vît si leur cervelet a des lamelles moins nombreuses et des sillons moins profonds que celui des hommes doués de toute leur intelligence.» (Note à page 481 du chapitre de De Saussure cité à la Note 44.)

${ }^{46}$ Ms. 77, c. $79 \mathrm{v}$.

47 Lettre de Malacarne à Bonnet du 20 Novembre 1787 (Ms. 40, c. 24r).

48 Page 33 de l'ouvrage $S u$ i gozzi rappelé par la Note 52.

49 Osservazioni sopra i Cretini della valle d'Aosta esposte con lettera al sig. Malacarne dal sig. Baile, Giornale scientifico letterario e delle arti di una Società filosofica di Torino 
raccolto e posto in ordine da Giovanni Antonio Giobert e dottor Carlo Giulio 4/1 (1789), p. 58-63.

Au juriste Lodovico Baylle (1764-1839), de Cagliari, auteur de cette lettre, datée Aoste le 26 Septembre 1789, Malacarne répondit de Turin le 9 Octobre suivant; voir Fanny Fontana-Zanco, Una lettera inedita di Vincenzo Malacarne sul gozzismo, Società Italiana di Storia delle Scienze Mediche e Naturali. Atti del IV. Congresso Nazionale, Roma 1933, Siena 1935 , p. 81-87.

50 Voir la Note 26.

51 Tentativo di Vincenzo Malacarne Saluzzese per discoprir le cagioni della stupidità endemica, e i mezzi di preservarne i fanciulli d'alcuni borghi della valle d'Aosta, e del Piemonte, Giornale scientifico letterario e delle arti ... 2/suppl. (1789), p. 331-352.

${ }^{52}$ L'ouvrage est dédié au comte Francesco Baldassarre di Perrone.

${ }^{53}$ Accompagné d'une table avec deux figures qui représentent l'anomalie cranienne en section sagittale médiane et sur la surface externe inférieure du crâne (où il semble de reconnaître aussi une fusion atlo-occipitale). On observe de toutes façons «eine sehr beträchtliche Vertiefung » (p. 34), c'est-à-dire l'impression basilaire.

${ }^{54}$ Voir la Note 29.

${ }^{55}$ Les frères Wenzel avaient étudié la question pendant une bonne dizaine d'années, en prenant également contact avec Frank. Celui-ci, «unser großer Lehrer», était alors à Pavie et leur fit connaître l'Essai de Fodéré (p. IX).

${ }^{56}$ François-Emanuel Foderé, Essai sur le goûtre et le crétinage où l'on recherche particulièrement quelles sont les causes de ces deux maladies des habitants des vallées, et quels sont les moyens physiques et moraux qu'il convient d'employer pour s'en préserver entièrement à l'avenir, Turin 1792 ; et traduction allemande, Berlin 1796.

En plus de l'Essai, Fodéré est également l'auteur du Traité du gôttre et du crétinisme, Paris 1800 (An VIII ${ }^{\mathrm{e}}$ ).

${ }^{57}$ Claude-Georges Collet, Un épisode obscur de la vie du docteur Fodéré, Revue de Savoie 10 (1957), p. 182-194 et table.

${ }^{58}$ Page 106.

59 Rudolf Virchow, Zur Entwicklungsgeschichte des Cretinismus und der Schädeldeformitäten, Gesammelte Abhandlungen zur wissenschaftlichen Medicin, Frankfurt a.M. 1856, p. 969-997. Deux études similaires de Virchow précèdent cet ouvrage, aussi bien chronologiquement que dans la pagination des Abhandlungen (p. 891-939 et p. 939-969).

${ }^{60}$ Voir l'ouvrage de Virchow cité à la Note 24. Parmi les allusions sporadiques à l'impression basilaire antérieures à cet ouvrage ressort la note de Joseph-Barnard Davis (1801-1881) : Sur les déformations plastiques du crâne, Mémoires de la Société d'Anthropologie de Paris 1 (1860/63), p. 379-390 et Planches VIII-IX.

${ }^{61}$ Rudolf Virchow, Untersuchungen über die Entwickelung des Schädelgrundes im gesunden und krankhaften Zustande und über den Einflu $\beta$ derselben auf Schädelform, Gesichtsbildung und Gehirnbau, Berlin 1857; Knochenwachstum und Schädelformen, mit besonderer Rücksicht auf Cretinismus, [Virchow's] Archiv für pathologische Anatomie und Physiologie und für klinische Medicin 13 (1858), p. 323-357.

${ }^{62}$ Représenté en entier à la page 976 de l'ouvrage déjà cité à la Note 59. La section sagittale de son crâne est reproduite à la figure 7 de la table IV de l'ouvrage de Virchow 1857 cité ici à la Note 61. 
${ }^{63}$ Wilhelm Weygandt, Über Virchow's Cretinentheorie, Neurologisches Centralblatt 23 (1904), p. 290-302, 352-361, 394-405.

${ }^{64}$ Pour le comportement de la base du crâne dans le crétinisme, voir la monographie désormais classique de Frédéric de Quervain et Carl Wegelin, Der endemische Kretinismus (= Pathologie und Klinik in Einzeldarstellungen, Band VII), Berlin/Wien 1936, p. 41 et p. 120-127, et figures 86 (en haut), 89 et 90 .

${ }^{65}$ Paul F. Cranefield, The Discovery of Cretinism, Bulletin of the History of Medicine 36 (1962), p. 489-511 ; L'origine probable de l'introduction du mot «Crétin» dans la langue écrite. Un manuscrit de 1750 par le Comte de Maugiron, Gesnerus 19 (1962), p. 89-92.

\section{Summary}

Between 1778 and 1789 Charles Bonnet and Vincenzo Malacarne discussed the "seat of the soul". Bonnet assumed it to be found at the (still unknown) point of convergence of the roots of the sensory nerfs. Malacarne preferred the cerebellum. He had found in 3 feebleminded persons, dissected in 1775,1779 and 1782 , a pronounced hypoplasia of the cerebellum, combined with "Basilary impressions of the skull" (as Virchow later called this anomaly). These anatomical findings of Malacarne contributed to the "definitive discovery of the cretinism" (Cranefield), based mainly on the clinical-epidemiological observations of cretins in the alpine valleys by Haller and de Saussure. In 1856 Virchow eliminated basilar impressions from the picture of cretinism and inserted them into anthropological craniology. Virchow described as typical change in cretinism the shortening of the skull base. $\mathrm{He}$ explained it by premature spheno-occipital synostosis, later authors by prolonged spheno-occipital synchondrosis.

Prof. Dr. med. Luigi Belloni

Istituto di Storia della Medicina

Università degli Studi

Via Festa del Perdono, 7

I-20122 Milano 